Results Out of 77 transfusions, 40 (52\%) were collected during the working hours while $37(48 \%)$ were collected out of hours. Nearly (40)52\% patients were stable at the time of transfusion and 25(32\%) were unstable and 12(16\%) were not recoreded.Median gestation and weight was at birth was 28 +3 weeks and $1.14 \mathrm{~kg}$ respectively. Median Day of Life at transfusion was 22. Median $\mathrm{Hb}$ level before transfusion was 104.5.

Conclusion A significant proportion of out of hour's orders are collected 5 to $7 \mathrm{pm}$ - could there be time savings made to get these within the $5 \mathrm{pm}$ cut off.More than half times when patients got transfused out of hours were stable. Could there be introduction of new guidelines in terms transfusing treatment in stable patients. Out of hours transfusions carries risk of increased work load on staff and costs more if transfusion pack is not available in the hospital.

\section{P463 AUDIT ON OFC MEASUREMENT IN THE ROTUNDA HOSPITAL NICU}

Roisin Egan*, Sheiniz Giva, Adrienne Foran. Dept. of Neonatology, The Rotunda Hospital, Dublin 1, Ireland

\subsection{6/archdischild-2019-epa.799}

Introduction Measurement of the occipital frontal circumference (OFC) is a quick and non-invasive method of monitoring head growth. Although it measures skull size, it also reflects overall brain volume and has been described as a 'widely used proxy of neural growth and brain size'. There is mounting evidence to suggest that suboptimal post natal head growth in term and preterm neonates is associated with neurodevelopmental delay. This is likely because of changes that occur during the 'critical' neonatal period ${ }^{[4]}$. Thus, serial OFC measurement not only has diagnostic but also prognostic significance.

Measurement is performed by placing a tape above the ears and midway between the forehead and the hairline to the occipital prominence. The first measurement should be performed at 36-48 hours after delivery to allow for resolution of oedema and moulding.

Methodology This review audit involved a spot review of 33 inpatient charts at the Rotunda Hospital NICU.

Exclusion criteria included patient instability at time of measurement and palliation.

The guideline of weekly OFC measurements for inpatient neonates was used as standard of care. OFC was plotted on sex specific growth charts.

Results 32 of the 33 patients met the inclusion criteria. Of these, $\mathrm{n}=11(34.4 \%)$ were very preterm $(<31+6$ weeks), $\mathrm{n}=9 \quad(28.1 \%)$ late preterm $(32-36+6$ weeks $)$ and $\mathrm{n}=12$ $(37.5 \%)$ were term (>37 weeks). 50\% $(n=16)$ did not meet the weekly OFC measurement standard. Of these, 50\% $(n=8)$ were on CPAP or BiPAP at time of missed measurement. Whilst the majority $(n=11)$ missed only one measurement, 3 missed two and 2 missed three; 4 of these were extremely premature $(<28$ weeks) and 2 had known abnormalities on cranial ultrasound. Four babies had no recorded OFC measurement. Furthermore the intervals between measurements varied, ranging from 1-14 days.

Conclusion The above audit demonstrates lack of adherence to our NICU guideline of weekly OFC measurements in 50\% of our inpatients.
Recommendations We recommend identification of those who are at high risk for missed measurements (e.g: infants on $\mathrm{CPAP} / \mathrm{BiPAP}$ ) and the implementation of strategies for opportunistic measurements in this cohort. As part of the NICU team, it would be beneficial to involve other carers in this task while performing other routine measurements such as weight checks. Dissemination of the results of this audit will be important in increasing awareness and the need to reaudit once new systems have been put in place.

\section{P464 BREASTFEEDING IN VERY LOW BIRTH WEIGHT}

Hacer Ergin*, Nuran Özçiftçi Ertuğral, Özmert Ma Özdemir, Ceren Çirali. Pamukkale University Faculty of Medicine Department of Neonatology, Denizli, Turkey

\subsection{6/archdischild-2019-epa.800}

Introduction Available research has shown that breastfeeding reduces the frequencies of sepsis, necrotizing enterocolitis, retinopathy of prematurity, and bronchopulmonary dysplasia (BPD), shortens reaching full enteral feeding and hospital stay, and improves neurodevelopment. Although sucking from breast prolongs breastfeeding duration in very low birth weight (VLBW, $\leq 1500 \mathrm{~g}$ ) infants, breastfeeding prevalence are still below the target levels.

Methods According to unit protocol, all newborns are fed with their mothers' milk if there is no medical obstacle. Breast milk is given via orogastric tube, bottle or cup to the infants who can not suck from the breast. Orogastric feeding is accelerated through kangaroo mother care, and the application of breast milk smell and taste. Breastfeeding is supported after discharge by educated nurses at outpatient clinic. Data of VLBW infants discharged from our hospital and referred to our outpatient clinic were collected from medical records and their mothers. Feeding practices recorded according to corrected age at discharge and postdischarge were classified in three categories: exclusive breast milk (EBM), exclusive formula, and mixed (a combination of breast milk and formula). Exclusive breast milk and mixed feeding were named as breastfeeding.

Results This study included 115 VLBW infants discharged from our hospital (88) and referred to outpatient clinic (27). No significant difference was found between discharged and referred groups in terms of birth weight $(1047 \pm 272$ vs 1046 $\pm 253 \mathrm{~g})$, gestational age $(28 \pm 2.9$ vs $28 \pm 1.5 \mathrm{w})$, male gender $(48.9 \%$ vs $51.9 \%)$, SGA rates at birth and on discharge $[(15.9 \%, 30.7 \%)$ vs $(7.4 \%, 40.7 \%)]$, moderate/severe BPD incidence $(23.9 \%$ vs $40.7 \%)$, hospitalization duration $(57 \pm 27$ vs $78.2 \pm 31.7 \mathrm{~d}$ ], and mother education (high school\% 48,9 vs $66.7 \%)(\mathrm{p}>0.05)$.

At discharge, EBM (63.7\% vs 18.5\%) and breastfeeding rates $(91.7 \%$ vs $77.8 \%)$ were significantly higher in discharged infants compared with referred infants $(p<0.05)$. EBM and breatfeeding rates at $0-6$ months were $65.4 \%$ and $100 \%$ for discharged infants, $38.4 \%$ and $84.5 \%$ for referred infants. Breastfeeding rates were $95.5 \%$ and $88.9 \%$ in discharged infants, $81.8 \%$ and $66.6 \%$ were in referred infants at 6-12 months and 12-24 months. BPD existence reduced EBM rates at discharge $(23 \%$ vs\%76.8) and $0-6$ months $(19.3 \%$ vs $35.3 \%)$ in discharged infants from our hospital $(\mathrm{p}<0.05)$.

Conclusion The policies supporting breastfeeding during hospitalization and after discharge increases breastfeeding rates in VLBW infants. 